\title{
Polyhydroxylated Phragmalins from the Fruit of a Chinese Mangrove Xylocarpus granatum
}

\author{
Yuan Zhou, $^{\dagger, \ddagger}$ Fan Cheng, ${ }^{\dagger, \ddagger}$ Jun Wu, ${ }^{\ddagger} * \mathrm{Kun} \mathrm{Zou}^{\dagger}$
}

${ }^{\dagger}$ Chemistry \& Life Science College, China Three Gorges University, 8 University Road, Yichang 443002, and ${ }^{\ddagger}$ Guangdong Key Laboratory of Marine Materia Medica, South China Sea Institute of Oceanology, Chinese Academy of Sciences, 164 West Xingang Road, Guangzhou 510301, P. R. China

\section{wwujun2003@yahoo.com}

Supporting Information: Diagnostic NOE correlations of compounds 2-3; HR-FAB-MS, ${ }^{1} \mathrm{H}$ and ${ }^{13} \mathrm{C}$ NMR spectra of xyloccensins $\mathrm{Y}, \mathrm{Z}_{1}$ and $\mathrm{Z}_{2}$ (1-3). 


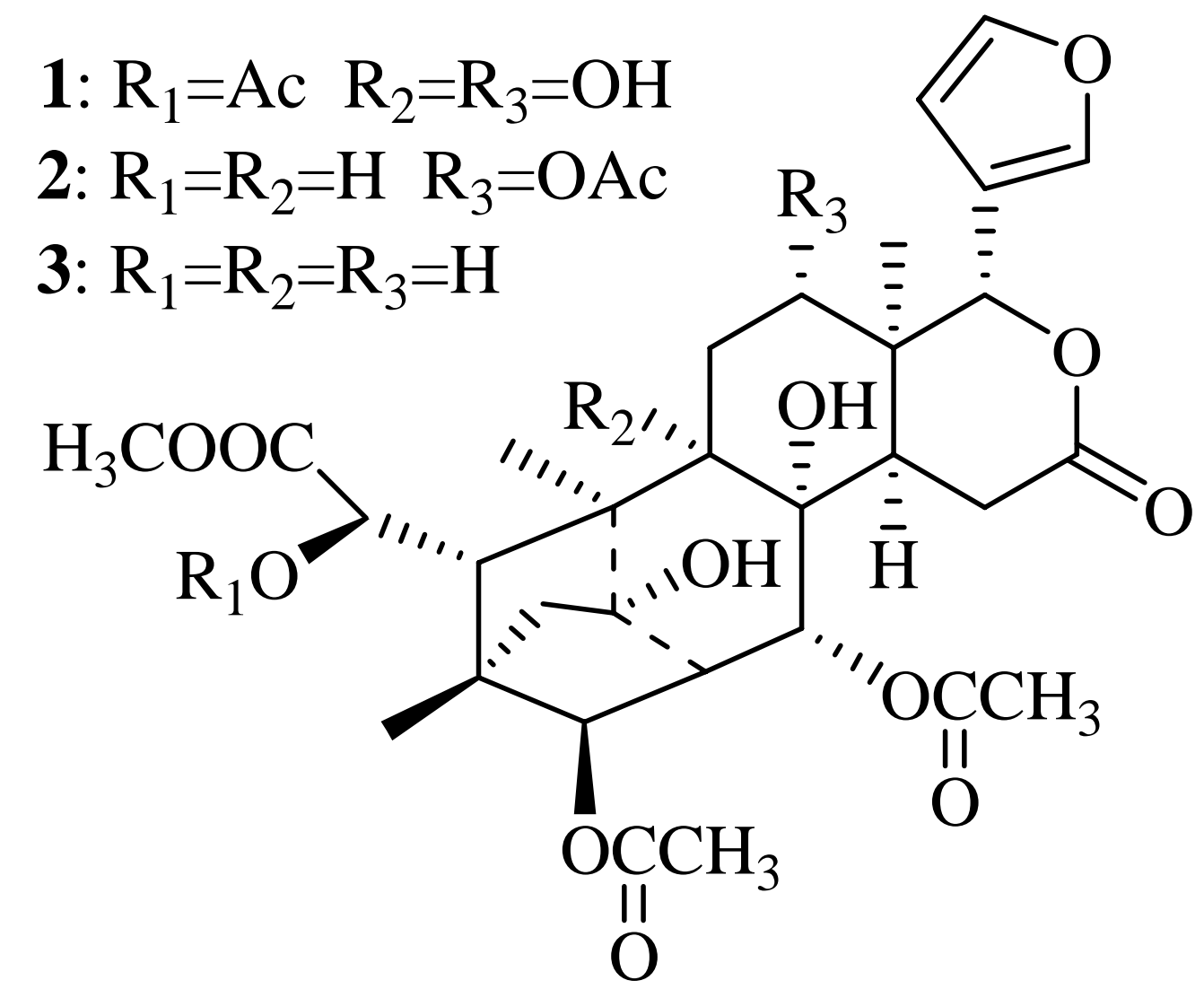

Structures of xyloccensins $\mathrm{Y}, \mathrm{Z}_{1}$ and $\mathrm{Z}_{2}$ (1-3) 


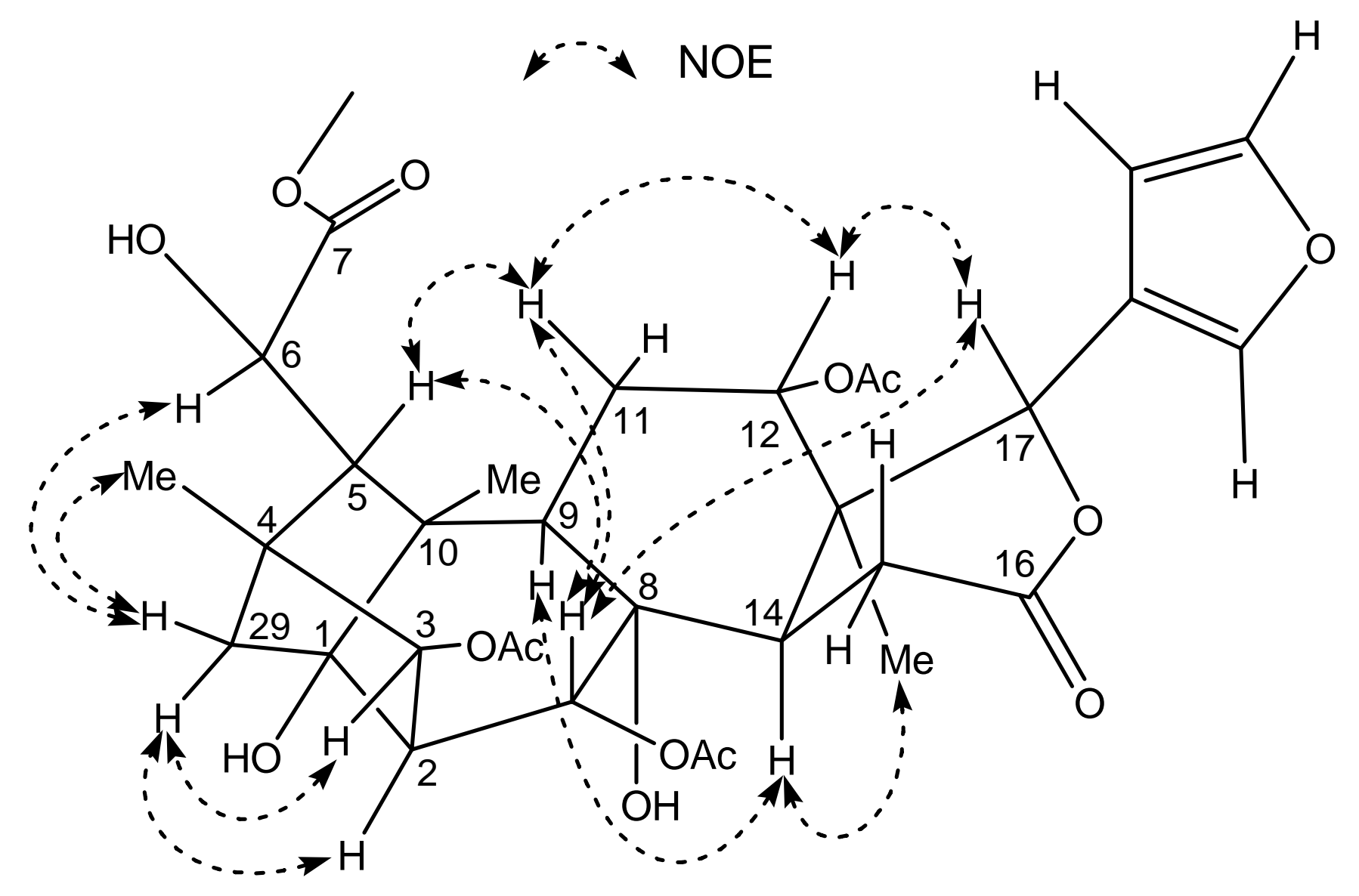

Figure 1. Diagnostic NOE correlations of compound 2. 


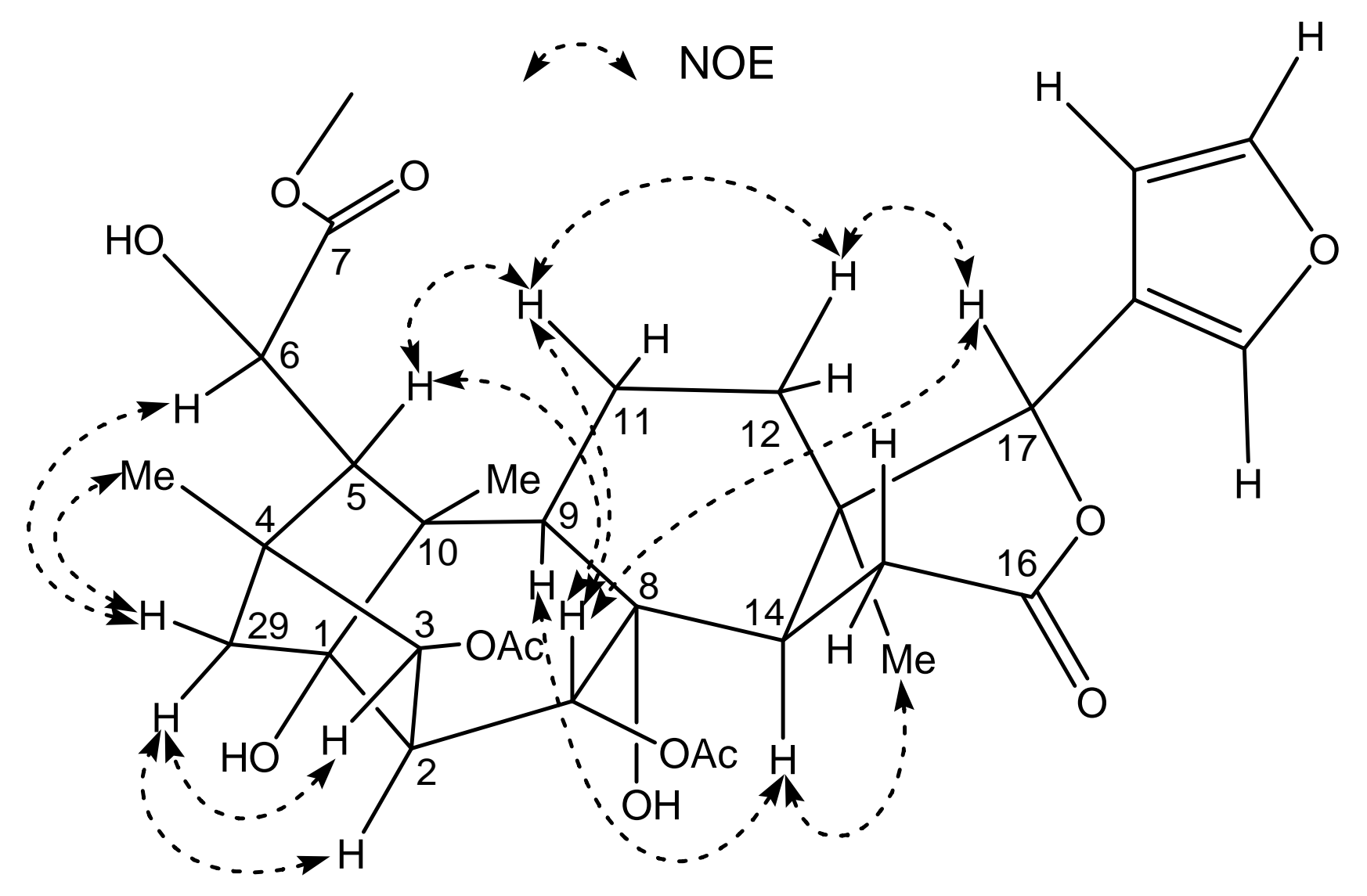

Figure 2. Diagnostic NOE correlations of compound 3. 
HR-FABMS of xyloccensin Y (1)

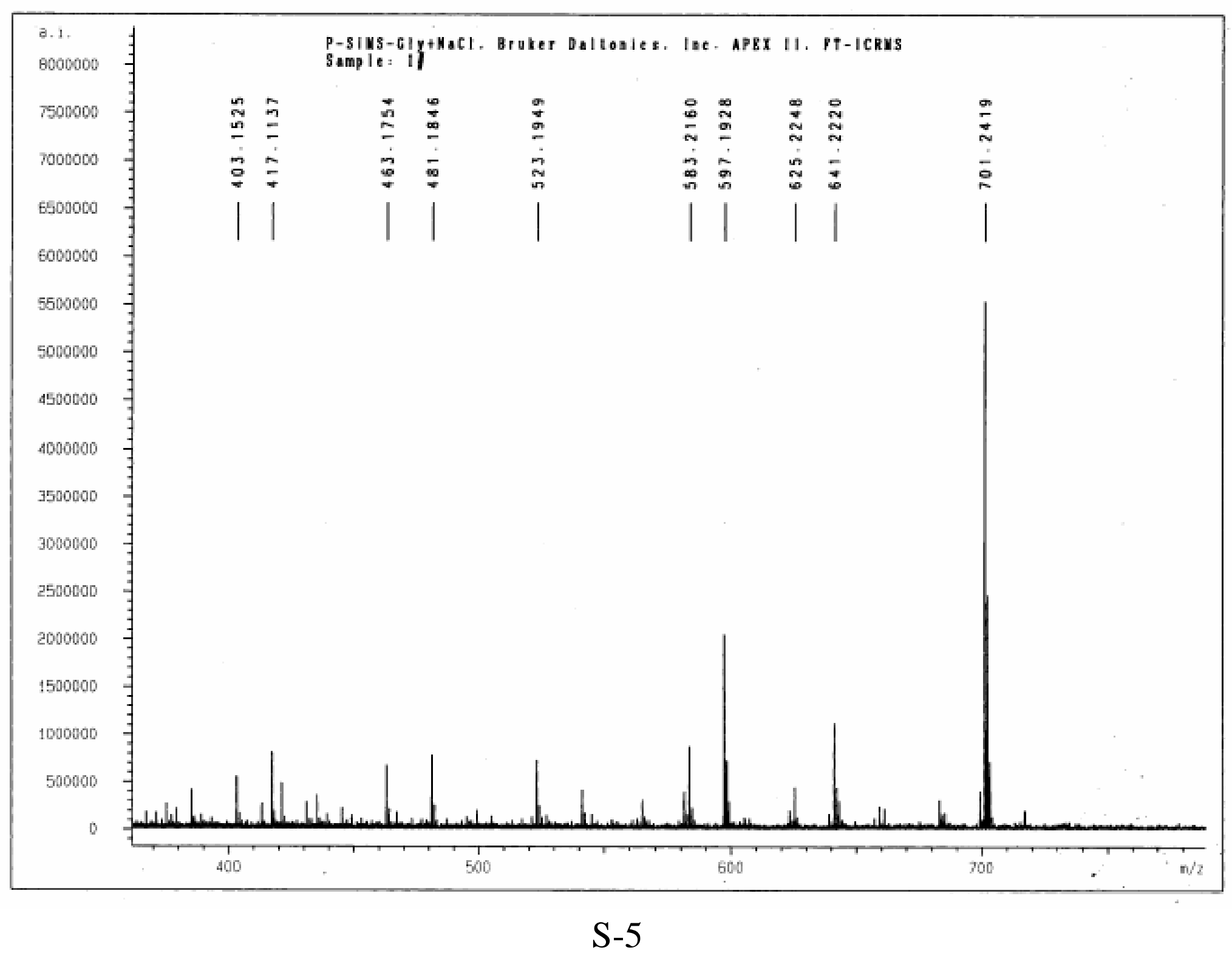


${ }^{1} \mathrm{H}$ NMR (500 MHz) of xyloccensin Y (1) in methanol- $d_{4}$

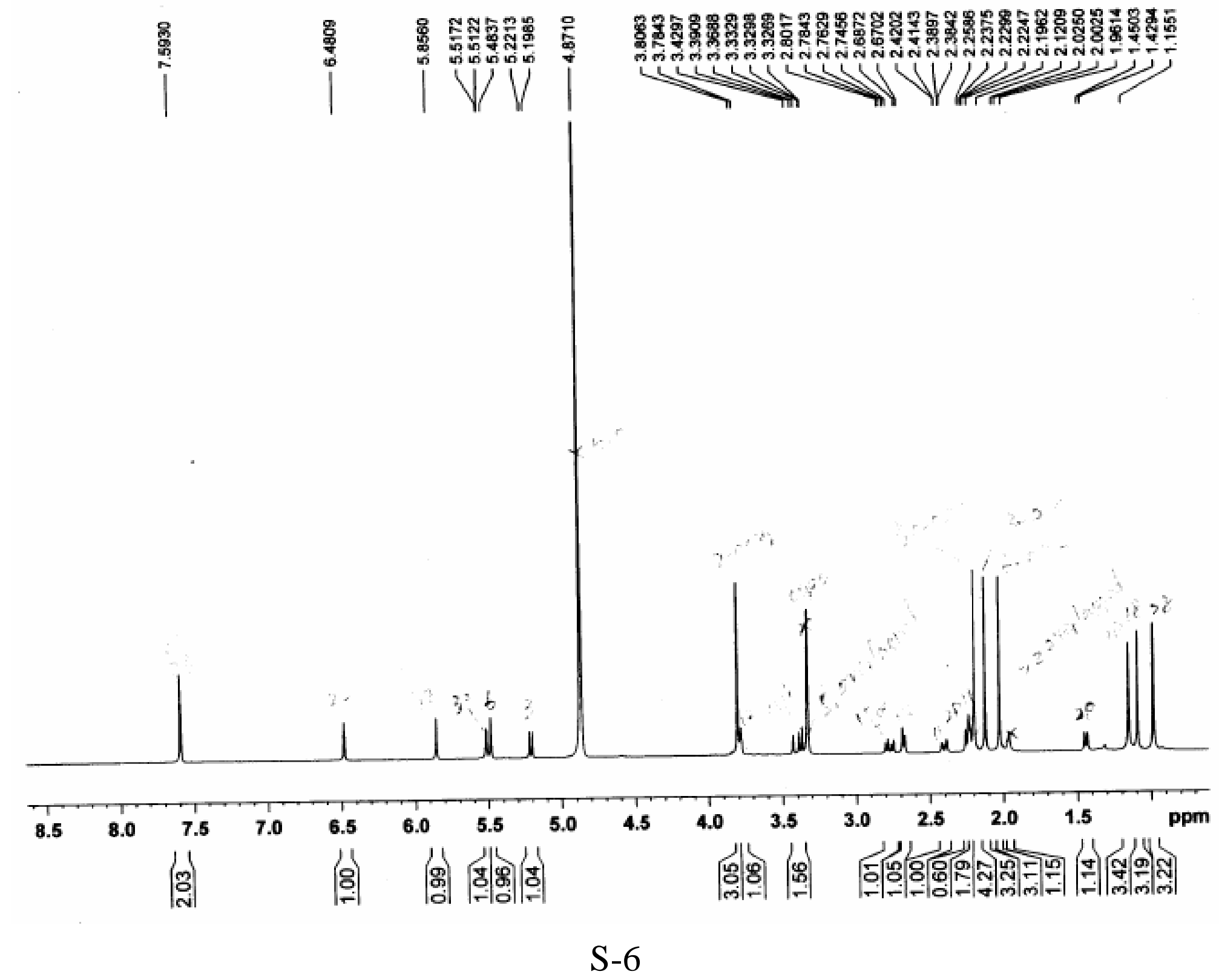


${ }^{13} \mathrm{C}$ NMR (125 MHz) and DEPT $135^{\circ}$ experiment of xyloccensin $\mathrm{Y}(\mathbf{1})$ in methanol- $d_{4}$

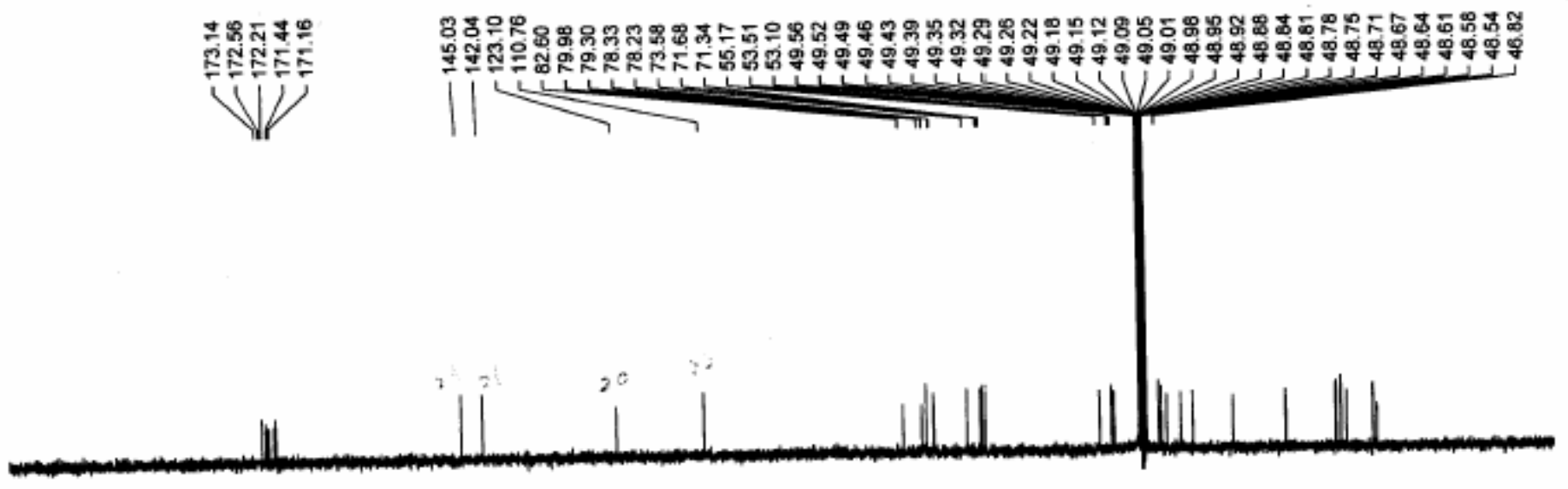

DEPT135 Spectrum of xyfE19B

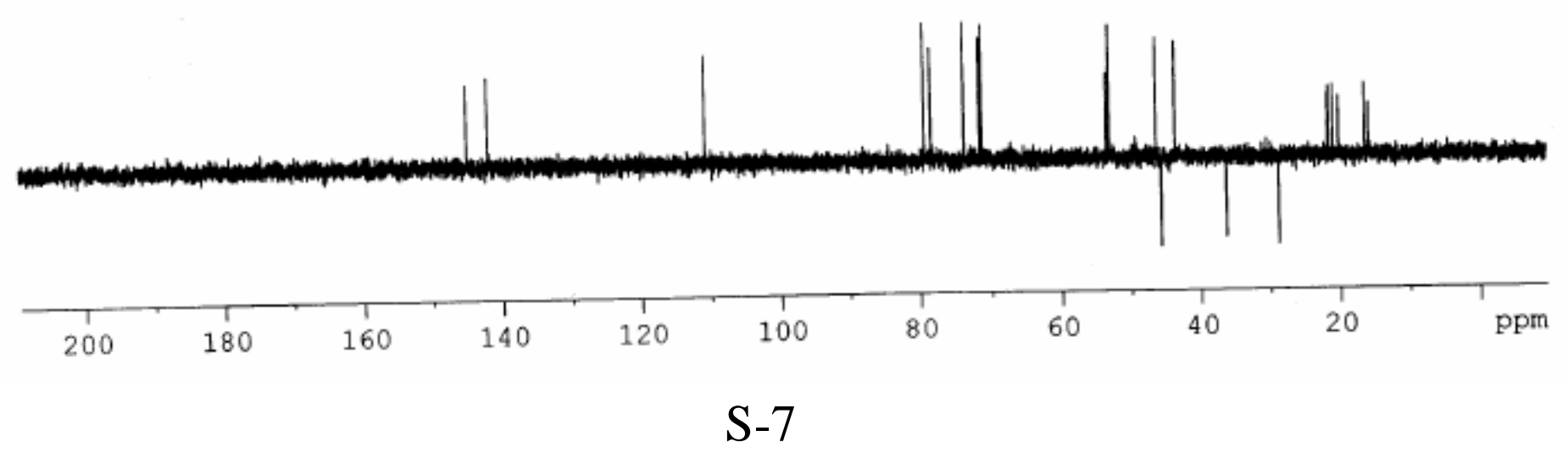


HR-FABMS of xyloccensin $Z_{1}$ (2)

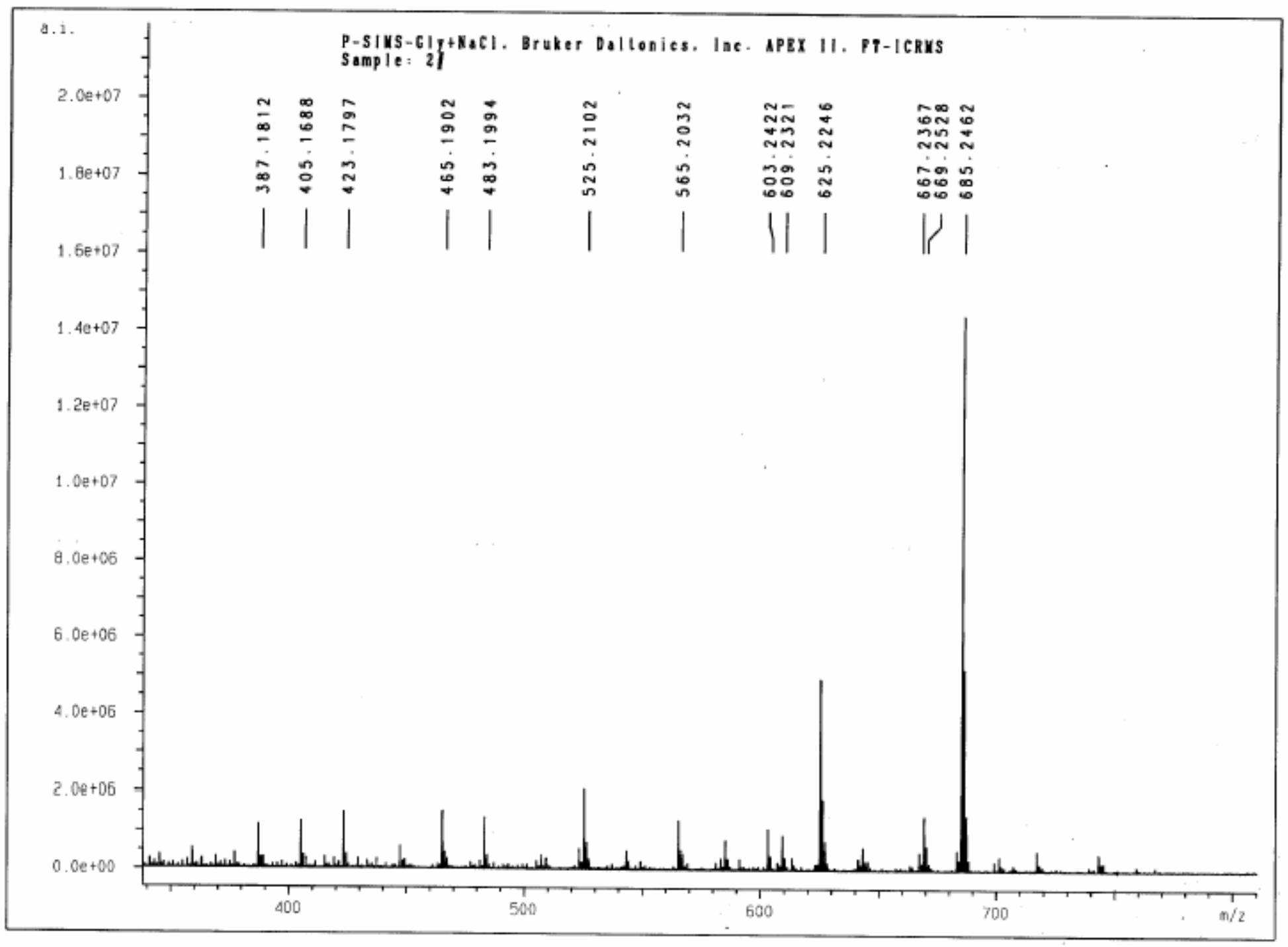


${ }^{1} \mathrm{H}$ NMR (500 MHz) of xyloccensin $\mathrm{Z}_{1}(2)$ in methanol- $d_{4}$

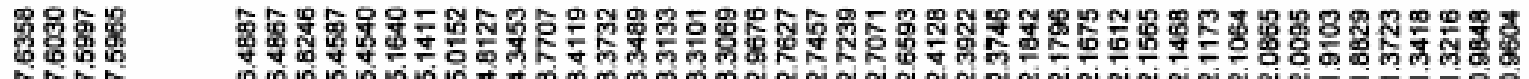

-

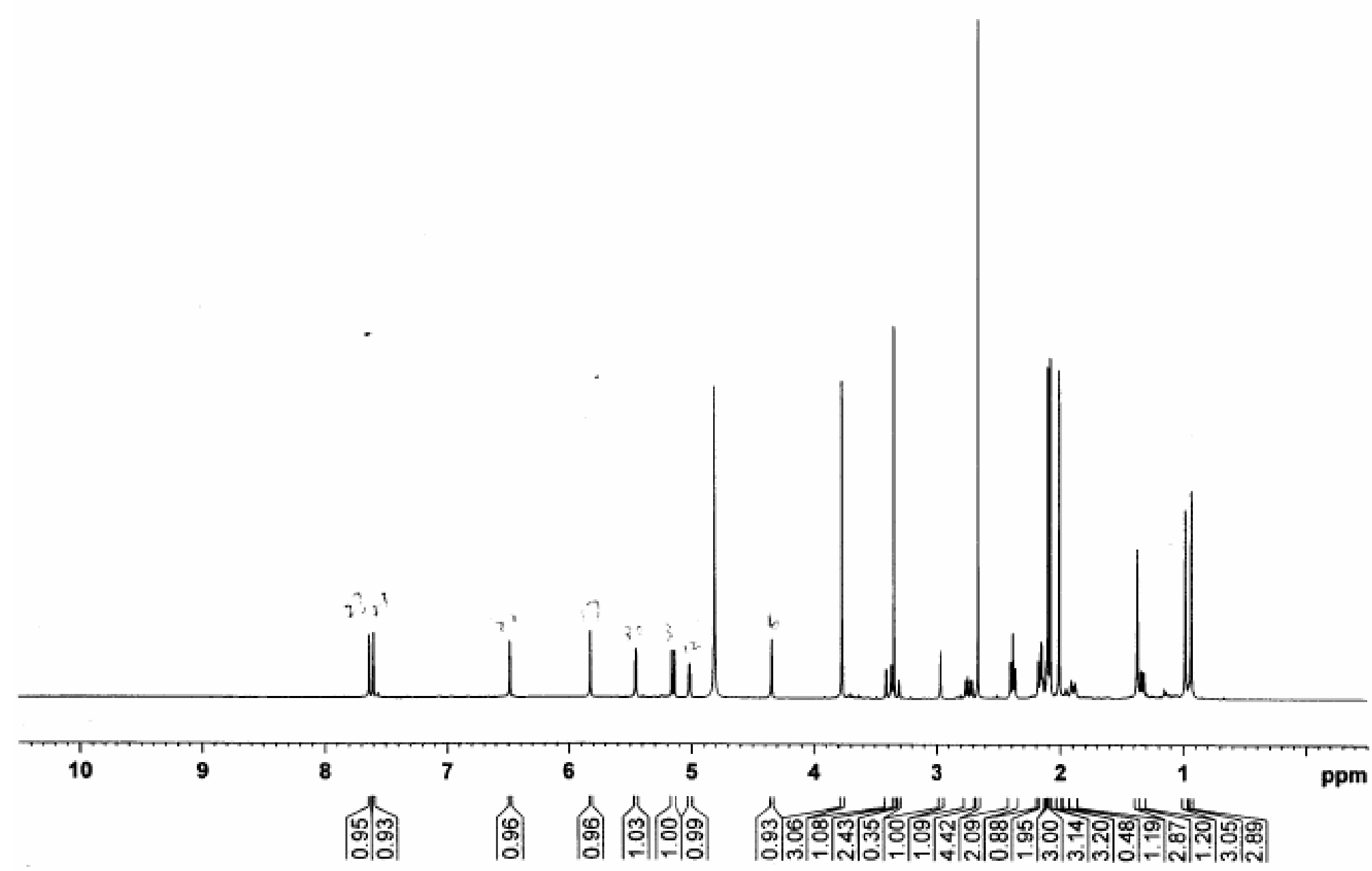

S-9 
${ }^{13} \mathrm{C}$ NMR (125 MHz) and DEPT $135^{\circ}$ experiment of xyloccensin $Z_{1}(2)$ in methanol- $d_{4}$

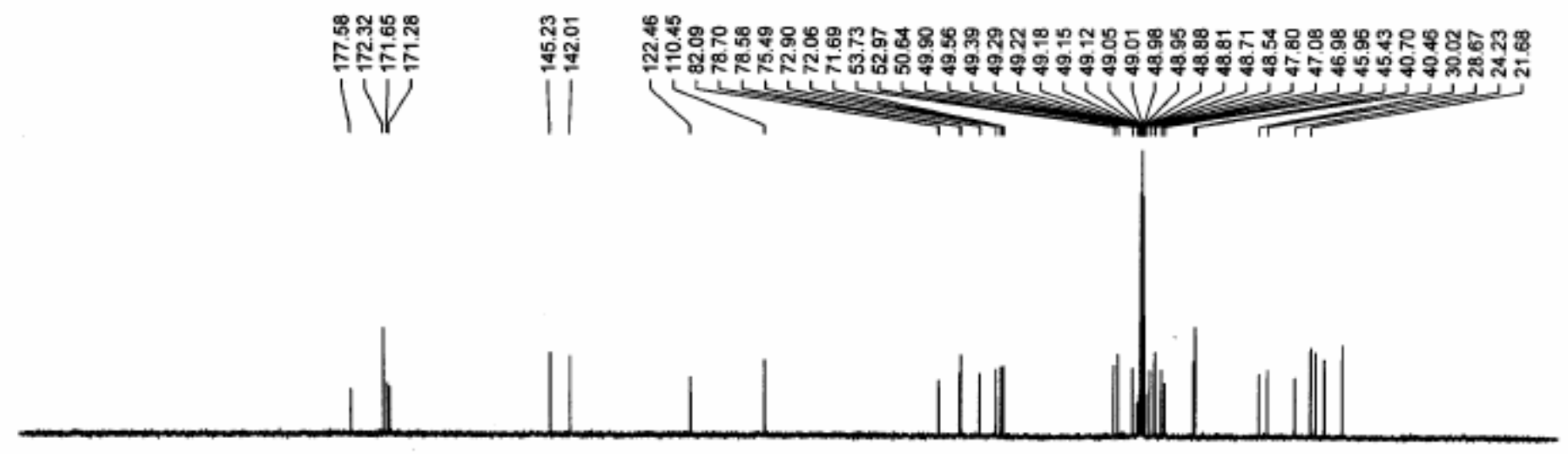

DEPT135 Spectrum of xyfE21
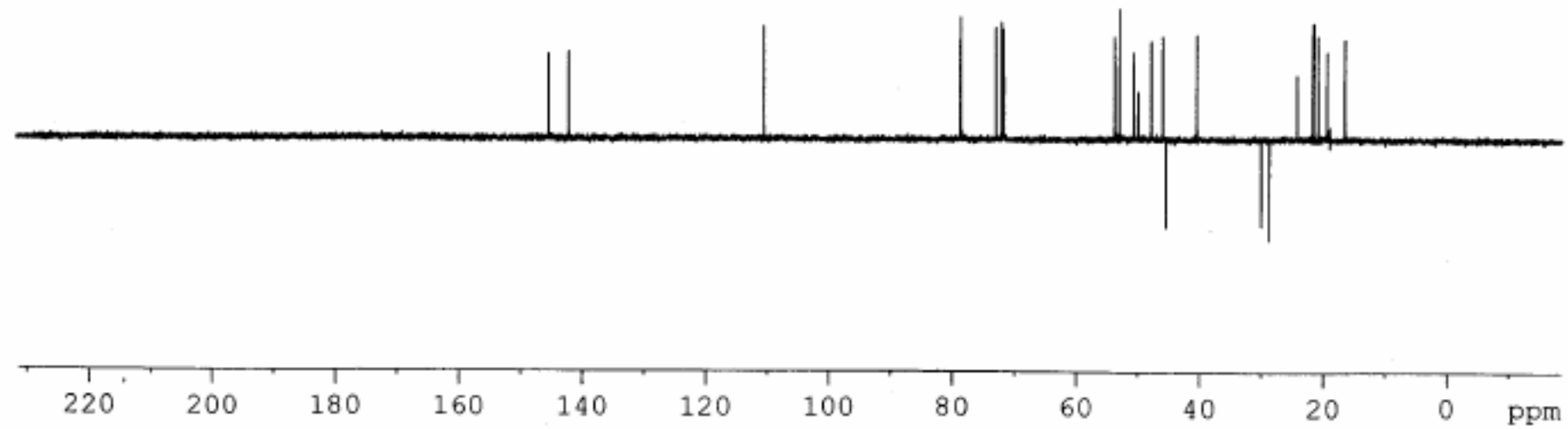

S-10 
HR-FABMS of xyloccensin $Z_{2}$ (3)

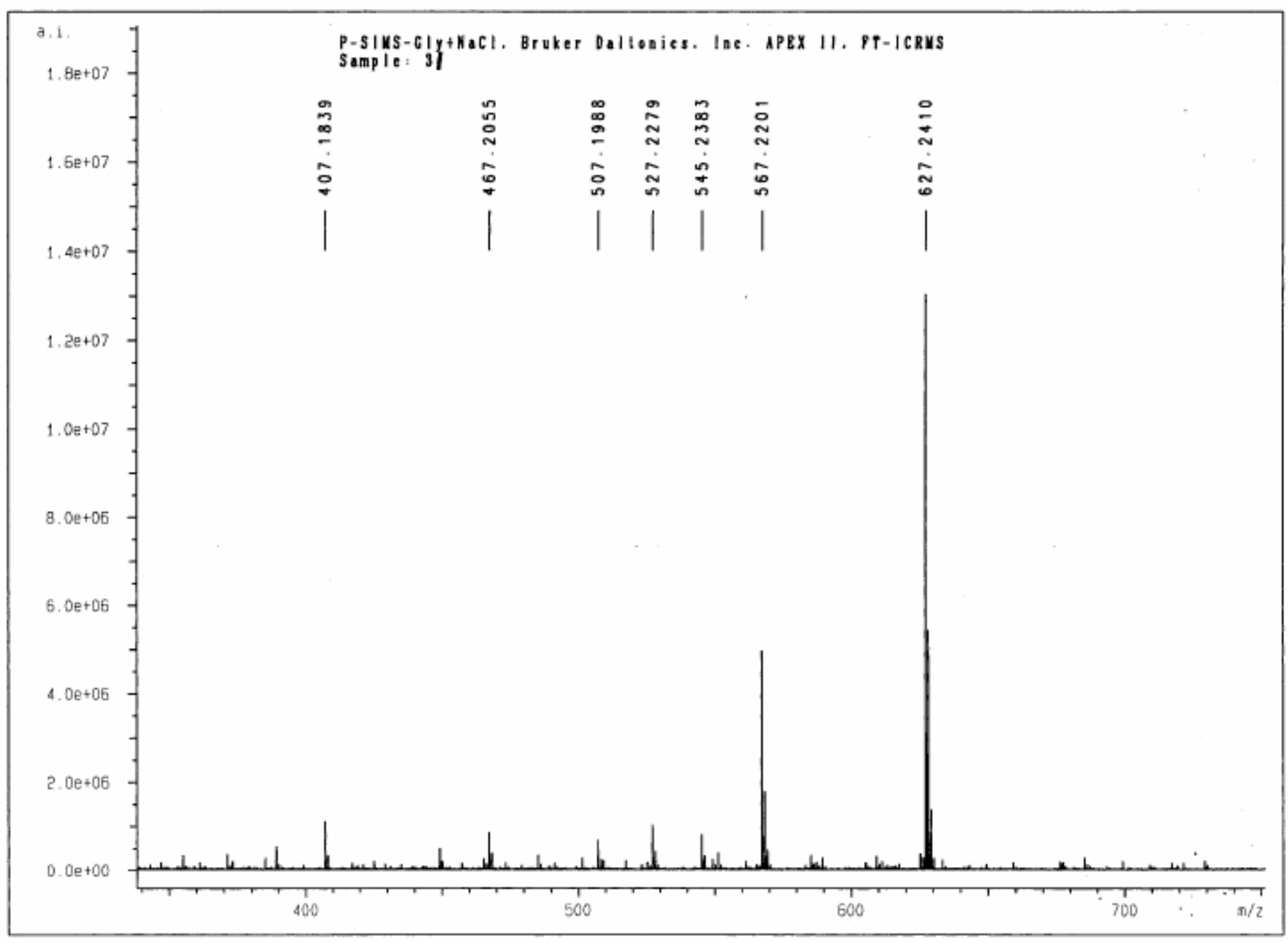

S-11 
${ }^{1} \mathrm{H}$ NMR (500 MHz) of xyloccensin $\mathrm{Z}_{2}(3)$ in methanol- $d_{4}$

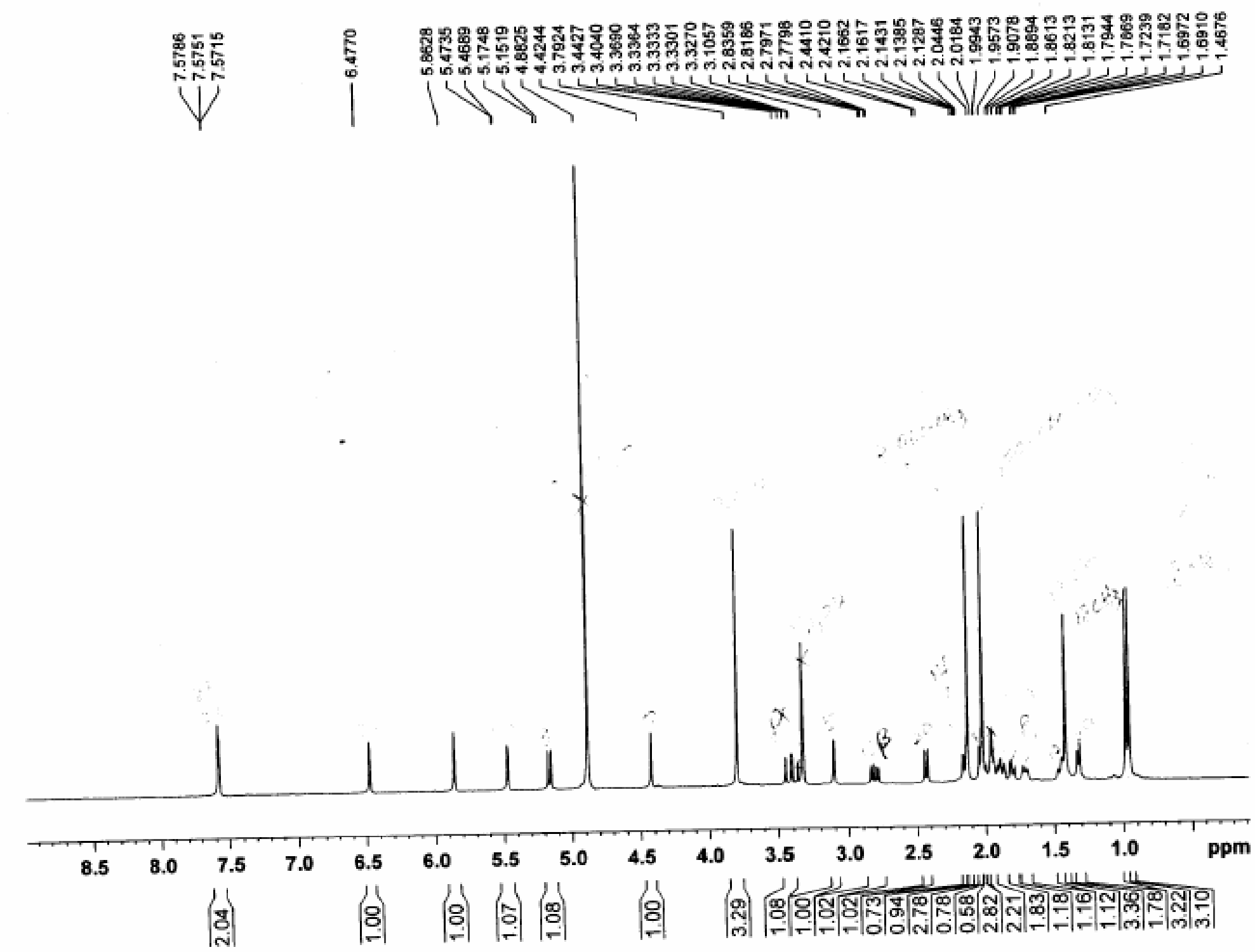


${ }^{13} \mathrm{C}$ NMR (125 MHz) and DEPT $135^{\circ}$ experiment of xyloccensin $Z_{2}(3)$ in methanol- $d_{4}$

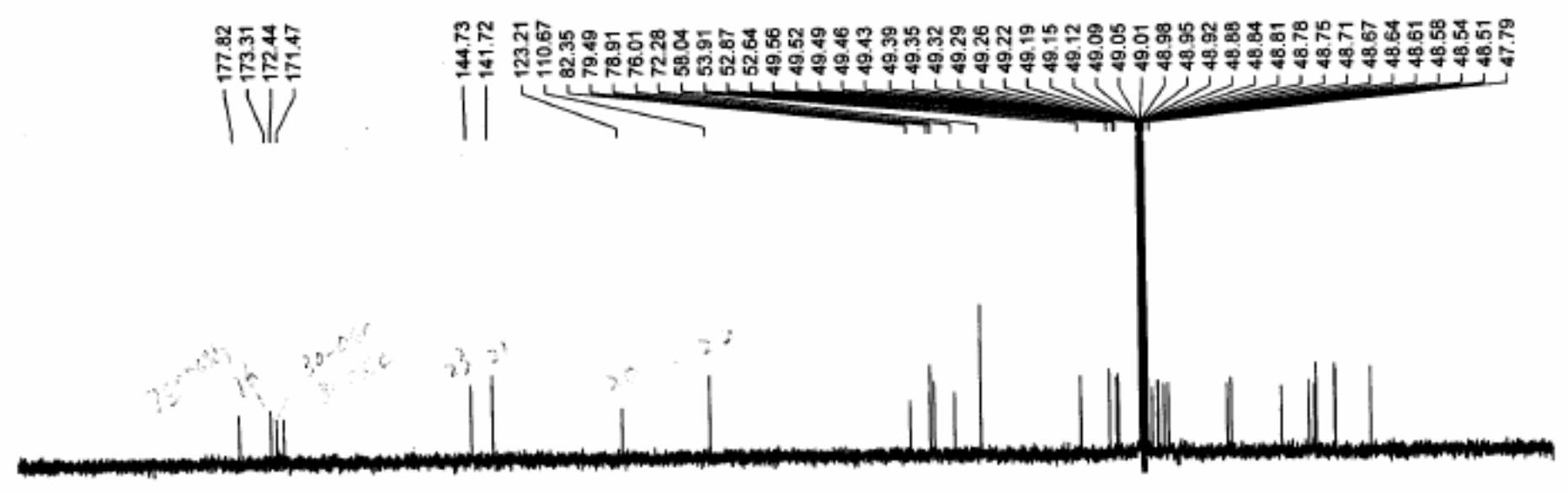

DEPT135 Spectrum

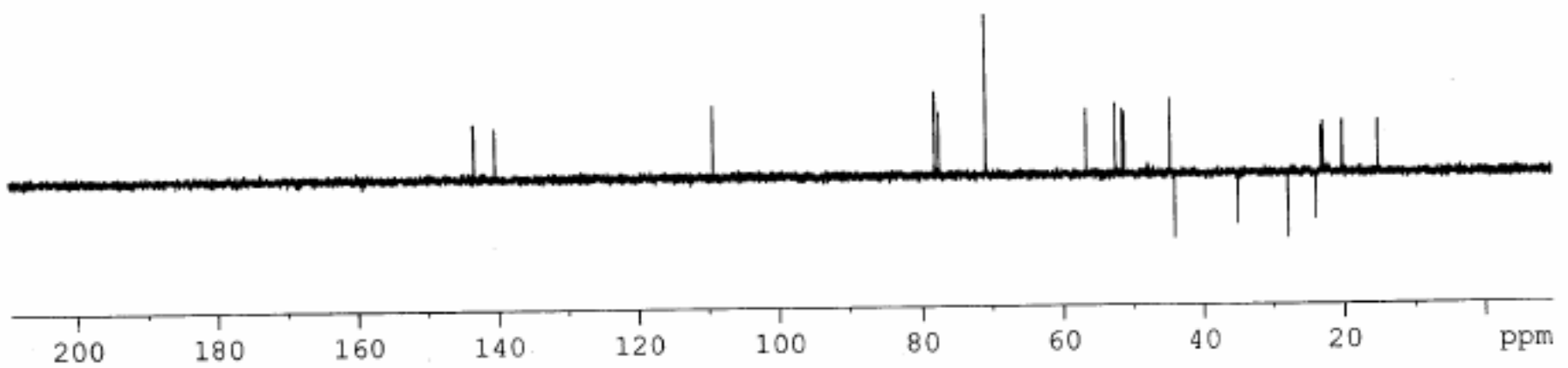

S-13 